\title{
Simple urine testing could avoid delay in the diagnosis of rapidly progressive glomerulonephritis
}

\author{
Matthew Howse, John Main
}

\begin{abstract}
Summary
Four cases of rapidly progressive glomerulonephritis are presented. In all four there was a two to three month delay between the onset of symptoms and appropriate diagnosis, referral and treatment. It is likely that simple stick testing of urine would have given a vital diagnostic clue and allowed earlier referral and treatment.
\end{abstract}

Keywords: urine testing, rapidly progressive glomerulonephritis, Wegener's granulomatosis

Rapidly progressive glomerulonephritis (RPGN) is the clinical manifestation of a varied group of inflammatory renal diseases. It is characterised by vague symptoms of ill health and rapid loss of renal function (over six weeks to six months). ${ }^{1}$ The most common specific diagnoses are Wegener's granulomatosis, microscopic polyangiitis, and anti-glomerular basement membrane disease (antiGBM disease). Urinalysis will almost always show at least $1+$ or more of both protein and blood. ${ }^{2}$ Although urine microscopy, by revealing red cell and granular casts, is more specific, simple stick testing is highly sensitive. RPGN is rare but prompt diagnosis and treatment is vitally important as the chances of renal salvage depend on the degree of damage at the time that treatment starts. ${ }^{3}$

We report four patients who were all unwell and attending doctors for at least two months before the diagnosis was made. Urinalysis could have accelerated the diagnosis and allowed earlier, more effective and less intensive treatment.

\section{Case reports}

\section{Case 1}

A 52-year-old man was seen over a 10-week period with nausea. His symptoms failed to respond to omeprazole. He began to lose weight and complained of an ammonia-like smell on his breath. Urinalysis then showed protein ++ , blood ++ , and serum creatinine $737 \mathrm{mmol} / 1$. $\mathrm{He}$ was admitted to hospital where a renal biopsy and serology confirmed a diagnosis of RPGN due to anti-GBM disease. He was treated with steroids, cyclophosphamide and plasma exchange and his creatinine initially improved to $400 \mathrm{mmol} / 1$. However, over the following months his renal function declined again and he became dialysis dependent.
Case 2

A 68-year-old man was treated for a viral illness. His initial symptoms were persistent nasal stuffiness and malaise. $\mathrm{He}$ was on enalapril for hypertension and a recent serum creatinine had been $130 \mathrm{mmol} / \mathrm{l}$. After two months his symptoms had failed to subside and on rechecking his creatinine was $999 \mathrm{mmol} / \mathrm{l}$. On admission to hospital he had large amounts of blood and protein in his urine. Renal biopsy showed a crescentic nephritis and serology supported a diagnosis of Wegener's granulomatosis. He was treated with steroids and immunosuppression and though dialysis dependent for the first week, recovered and had a serum creatinine of $200 \mathrm{mmol} / 1$ six months later.

Case 3

A 56-year-old man was seen over a threemonth period with malaise, joint pains and low mood. His symptoms failed to respond to lofepramine and ibuprofen and he developed episcleritis. When his biochemistry was checked he had a creatinine of $802 \mathrm{mmol} / \mathrm{l}$. Renal biopsy and serology confirmed the diagnosis of Wegener's granulomatosis. He was treated with steroids and immunosuppressives and also required dialysis for six weeks. Three months into treatment his creatinine had fallen to $330 \mathrm{mmol} / \mathrm{l}$. Over the next 36 months there was, however, an inexorable decline in his renal function and he returned to dialysis.

\section{Case 4}

A 52-year-old man had 'flu like symptoms and weight loss for one month despite three courses of antibiotics. At this point his general practitioner found an erythrocyte sedimentation rate of $110 \mathrm{~mm} / \mathrm{h}$ and serum creatinine of 105 $\mathrm{mmol} / \mathrm{l}$. He was referred to a general physician and seen one month later with his symptoms unchanged. Urinalysis was positive for blood and protein and serum creatinine was now $421 \mathrm{mmol} / 1$. A chest X-ray suggested a hilar mass. He was admitted one week later for bronchoscopy. Over the next three days he became pyrexial, anuric and developed pulmonary oedema. He was transferred to this renal unit with a creatinine of $820 \mathrm{mmol} / 1$. A diagnosis of Wegener's granulomatosis was made based on clinical features and serology. Steroids and immunosuppression were started and he required haemodialysis for two weeks. Six weeks later serum creatinine was 300 $\mathrm{mmol} / \mathrm{l}$. His progress was complicated by the 
development of a staphylococcal spinal abscess but 24 months later he was well with a serum creatinine of $200 \mathrm{mmol} / \mathrm{l}$.

\section{Discussion}

These cases highlight the delay which commonly occurs in diagnosing RPGN and its serious consequences. This delay may be due to a number of factors. Firstly, these diseases are rare, accounting for only $5 \%$ of patients with end-stage renal failure, ${ }^{4}$ so that general practitioners are unlikely to come across more than one case in their career.

Secondly, these illnesses often present with a variety of nonspecific but common symptoms which could reasonably be expected to be due to a self-limiting condition. These symptoms often mimic viral infections and do not obviously relate to any renal disease. Typically, those affected complain of tiredness, anorexia and generalised arthralgia. Those with Wegener's granulomatosis often have had nasal stuffiness and a bloody nasal discharge for weeks before presentation, and their speech may have an obvious nasal quality. They may have a characteristic skin rash, especially on the elbows and knees. ${ }^{5}$ Generalised malaise is also a feature of anti-GBM disease, and those with lung involvement (Goodpasture's syndrome) frequently have haemoptysis. ${ }^{6}$

Although simply observing the effect of time on such symptomatology is often helpful, we would suggest that, in any patient with an unexplained vague illness of greater than three weeks duration, investigations should include testing their urine for blood and protein. If these are positive the urine should be sent for culture and serum creatinine measured. If the culture is negative then an early renal opinion is mandatory. If the creatinine is already raised then we suggest telephoning rather than writing. While ancillary tests such as appropriate autoantibody titres, ie, antineutrophil cytoplasmic antibody (ANCA), anti-GBM antibodies, and antinuclear factor, may be helpful ${ }^{7}$ referral should not be delayed while the results are awaited.

1 Weber M. Rapidly progressive glomerulonephritis: recent advances in pathogenesis, diagnosis, and therapy. Clin Invest 1993; 71: 825-9.

2 Pusey CD, Rees AJ. Acute renal failure due to vasculitis and glomerulonephritis. In: Cameron J, Davison AM, Kerr DNS, Ritz E, eds, Oxford textbook of clinical nephrology. Oxford: Oxford University Press, 1992; pp 1060-76.

3 Heilman RL, Offord KP, Holley KE, Velosa JA. Analysis of risk factors for patient and renal survival in crescenteric glomerulonephritis. Am $\mathscr{f}$ Kidney Dis 1987; 9: 98-107.

4 Ochi RF, Couser WG. Idiopathic rapidly progressive glomerulonephritis. In: Glassock RJ, ed, Current therapy in nephrology and hypertension. Toronto: Becker PC Inc, 1987; pp 189-92.

5 Hoffman GS, Kerr GS, Leavitt RY, et al. Wegener granulomatosis: an analysis of 158 patients. Ann Intern Med 1992; 116: 488-98.
Thirdly it may not be fully appreciated that, in RPGN, blood and protein appear in the urine before serum creatinine rises. Indeed, $50 \%$ of renal functions can be lost before this is elevated, ${ }^{8}$ and a normal serum creatinine, as in case 2, does not exclude RPGN. The findings of proteinuria or haematuria are not specific for RPGN but are more sensitive than serum creatinine in the early stages of the disease.

Finally, the terminology is confusing for non-nephrologists. RPGN is a clinical syndrome which has numerous, but rare, causes. The histological equivalent is crescentic nephritis and sometimes the clinical and histological terms are inadvertently transposed. ${ }^{9}$ The situation is further confused by the array of serological tests involved (pANCA, cANCA, anti-GBM, etc). While they may be useful in supporting the final diagnosis they may obscure the importance of a test as simple as urinalysis. ANCA results may take several weeks to become available in some hospitals and meanwhile valuable renal function may be lost.

Delays in diagnosis are clearly not confined to our catchment area. The Stuart Strange Trust, a charitable self-help organisation, has recently highlighted this problem by sending letters to Members of Parliament and the Directors of Public Health. Although their original cause for concern was the availability of facilities for diagnosing and treating these conditions, they came to the conclusion that the fundamental problem is not the availability of tests but thinking of doing them in the first place. ${ }^{10}$

The benefits of sufficiently early treatment are so great (such as the prevention of endstage renal failure and the avoidance of emergency dialysis and its complications) that attempts must be made to increase awareness of these conditions. Obviously, only a small proportion of patients with vague symptoms will have RPGN, but early urinalysis in this group of patients seems more logical than the mass, infrequent testing of the entirely well.

6 Walker RG, Scheinkestel C, Becker GJ, et al. Clinical and morphological aspects of the management of crescentic antiglomerular basement membrane antibody (anti-GBM) nephritis / Goodpasture's syndrome. $Q \mathcal{F}$ Med 1985; 54: 75-89.

7 Falk R. ANCA-associated renal disease. Kidney Int 1990; 38: 998-1010.

8 Bannister KM, Field MF. Clinical physiology of the kidney: tests of renal function and structure. In: Weatherall DJ Ledingham JGG, Warrell DA, eds, Oxford textbook of medicine. Oxford: Oxford University Press, 1996; pp 310115.

9 Couser WG. Idiopathic rapidly progressive glomerulonephritis. Am f Nephrol 1982; 2: 57-69.

10 Stuart Strange Trust Newsletter, 7 October 1995. 